This item was submitted to Loughborough's Research Repository by the author.

Items in Figshare are protected by copyright, with all rights reserved, unless otherwise indicated.

\title{
Automatic liver vessel segmentation using 3D region growing and hybrid active contour model
}

PLEASE CITE THE PUBLISHED VERSION

https://doi.org/10.1016/j.compbiomed.2018.04.014

\section{PUBLISHER}

(C) Elsevier

\section{VERSION}

AM (Accepted Manuscript)

\section{PUBLISHER STATEMENT}

This work is made available according to the conditions of the Creative Commons Attribution-NonCommercialNoDerivatives 4.0 International (CC BY-NC-ND 4.0) licence. Full details of this licence are available at: https://creativecommons.org/licenses/by-nc-nd/4.0/

\section{LICENCE}

CC BY-NC-ND 4.0

\section{REPOSITORY RECORD}

Zeng, Ye-zhan, Sheng-hui Liao, Ping Tang, Yu-qian Zhao, Miao Liao, Yan Chen, and Yi-xiong Liang. 2019. "Automatic Liver Vessel Segmentation Using 3D Region Growing and Hybrid Active Contour Model". figshare. https://hdl.handle.net/2134/33050. 


\title{
Automatic Liver Vessel Segmentation Using 3D Region Growing \\ and
}

\section{Hybrid Active Contour Model}

\author{
Ye-zhan Zeng ${ }^{a, b}$, Sheng-hui Liao ${ }^{a}{ }^{*}$, Ping Tang ${ }^{a, b}$, Yu-qian Zhao ${ }^{a, b}{ }^{*}$, Miao Liao ${ }^{c}$, Yan Chen ${ }^{d}$, Yi-xiong Liang ${ }^{a}$ \\ a School of Information Science and Engineering, Central South University, Changsha 410083, China \\ ${ }^{b}$ Department of Biomedical Engineering, Central South University, Changsha 410083, China \\ c School of Computer Science and Engineering, Hunan University of Science and Technology, Xiangtan 411201, China \\ ${ }^{d}$ Applied Vision Research Centre, Loughborough University, Loughborough, UK
}

\section{*Contact: Ish@csu.edu.cn; zyq@csu.edu.cn}

\begin{abstract}
This paper proposes a new automatic method for liver vessel segmentation by exploiting intensity and shape constraints of 3D vessels. The core of the proposed method is to apply two different strategies: 3D region growing facilitated by bi-Gaussian filter for thin vessel segmentation, and hybrid active contour model combined with K-means clustering for thick vessel segmentation. They are then integrated to generate final segmentation results. The proposed method is validated on abdominal computed tomography angiography (CTA) images, and obtains an average accuracy, sensitivity, specificity, Dice, Jaccard, and RMSD of $98.2 \%, 68.3 \%, 99.2 \%, 73.0 \%, 66.1 \%$, and $2.56 \mathrm{~mm}$, respectively. Experimental results show that our method is capable of segmenting complex liver vessels with more continuous and complete thin vessel details, and outperforms several existing 3D vessel segmentation algorithms.
\end{abstract}

Key words: Bi-Gaussian filter, 3D region growing, Hybrid active contour model, Liver vessel segmentation

\section{Introduction}

Liver vessel segmentation from abdominal computed tomography angiography (CTA) images is an essential and key step for liver-related disease diagnosis and therapy, liver transplantation, as well as 
liver-tumor resection[1]. However, influenced by the significant noise, partial volume effects, various vessel sizes, and especially by inhomogeneous intensity distribution and highly ramified branches, accurate segmentation of liver vessels from abdominal CTA images is a challenging task. In fact, accurate vessel segmentation in clinical practice usually relies on the manual delineation by radiologists on each CTA slice, which is extremely tedious and time-consuming. Thus, automatic or semi-automatic segmentation of liver vessels gains increasing attention.

Usually, three-dimensional (3D) vessels in medical images can be modeled as tubular structures, whose cross-sections can be described as asymmetrical circular or elliptic shape [2]. Based on this geometric structure, many efforts have been made for 3D vessel segmentation including active contour model/level set-based [3-8] and graph cuts-based techniques [9-10].

Shang et al. [3] described a region competition-based active contour mode exploiting a Gaussian mixture model for the segmentation of thick vessels, and introduced a vascular vector field to drive the active contour into thin and weak vessels. This method is effective in various vessel segmentation. Cheng et al. [4] developed an active contour framework for vessel segmentation. It starts with an axis tracking technique to detect vessel cross-section, and then applies a B-snake model for boundary refinement. With the shape and size constrains on the cross-section of vessels, this method is able to accurately segment healthy vessels. In [5], Woźniak et al. employed a multi-scale Frangi filter and an improved level set method for 3D vessel tree segmentation. In their method, a vessel force generated by Frangi filter is introduced to the level set formation, which enables the contour to move toward vessel boundaries actively and finds more vessel branches. Lu et al. [6] proposed a variational level set method for liver vessel segmentation, which first adopts a K-means clustering to select seed points and extracts vessel features from these seed points, and then uses a variational level set combined with bias correction for vessel segmentation. Zhao et al. [7] adopted a region-based active contour model combined with a phase-field method and statistical information for vessel network extraction. This method shows satisfying segmenting results when the vessel regions and background are separated in maximum intensity projection (MIP) image. Based on an analysis of the intensity distribution of magnetic resonance angiography (MRA) images, Gao et al. [8] constructed a statistical model and applied a fast level set method combined with region and gradient information to segment cerebrovascular vessels. Generally, the active contour model can provide a satisfying result for the high-contrast image, but the performance may be limited when handling the object with serious inhomogeneity. 
Recently, graph cuts algorithm has been widely applied in medical image segmentation for its efficient computation to the global optimal solution. Bauer et al. [9] applied a medialness filter together with height ridge traversal method to generate vessel prior which is then incorporated into graph cuts for the segmentation of 3D vessels. Their method was verified to be robust to separate different vessel systems and effective to determine the surface of tubular structures. Esneault et al. [10] introduced a 3D geometrical moment-based detector of cylindrical shapes to detect tubular structure, followed by graph cuts for liver vessel segmentation, and their experiments on the medical CTA images show a good segmenting ability. Sangsefidi et al. [11] defined a balanced data term of graph cuts to improve segmentation of hepatic vessels. They used vessel centerlines to estimate the local data term, which can be applied to balance the total energy in small vessels and low-contrast regions. In [12], the graph cuts is coupled with oriented flux measure and height ridge traversal to perform the segmentation and identification of liver vessels. This method works well on the high-contrast CTA images, and the effective extraction of centerline made it adaptive to various vessel structures. The drawbacks of [12] are its long computing time and sensitivity to image contrast.

Besides, Law and Chung [13] proposed a physics-based deformable surface model for vessel segmentation by reducing the influence of intensity contrast fluctuations along blood vessels. An extreme learning machine (ELM) algorithm was employed by Zeng et al. [14] for liver vessel segmentation beginning with four 3D vessel filters including Sato, Frangi, medialness, and strain energy to extract vessel features, followed by an ELM to recognize liver vessels from the background. Considering the equal distances from vessel centerline to the edges, Foruzan et al. [15] defined a medial-axis enhancement filter according to the eigenvalues of the Hessian matrix, and applied a dynamic thresholding for vessel segmentation from the noisy CT image. Yang et al. [16] firstly applied empirical threshold intervals to extract seed points, and then preformed a region growing method integrated in insight segmentation and registration toolkit (ITK) for vessel segmentation. This method can separate hepatic and port veins, however, requires manual selection of optimal segmentation result from six vessel candidates generated from different threshold intervals. Marcan et al. [17] adopted the Frangi filter and region growing to segment hepatic vessels, which receives an average sensitivity of $93.68 \%$ on magnetic resonance images (MRI).

These aforementioned methods receive satisfactory segmentation results for most of $3 D$ vessels; however, to the best of our knowledge, few methods consider the elongated and inhomogeneous 
structures of thin vessels with low contrast. In this paper, we propose an automatic liver vessel segmentation method using 3D region growing and hybrid active contour model. First, an anisotropic diffusion filter is used to remove noise while preserving vessel boundaries. Then, according to vessel geometrical structure, a bi-Gaussian filter together with a 3D region growing is adopted for thin-vessel segmentation. Next, based on an analysis of intensity distribution for liver vessels, a combination of K-means clustering and hybrid active contour model is used for automatic segmentation of thick vessels. Finally, both of the thin and thick vessels are integrated to generate the final liver vessel segmentation result. A flowchart of our method is shown in Fig. 1.

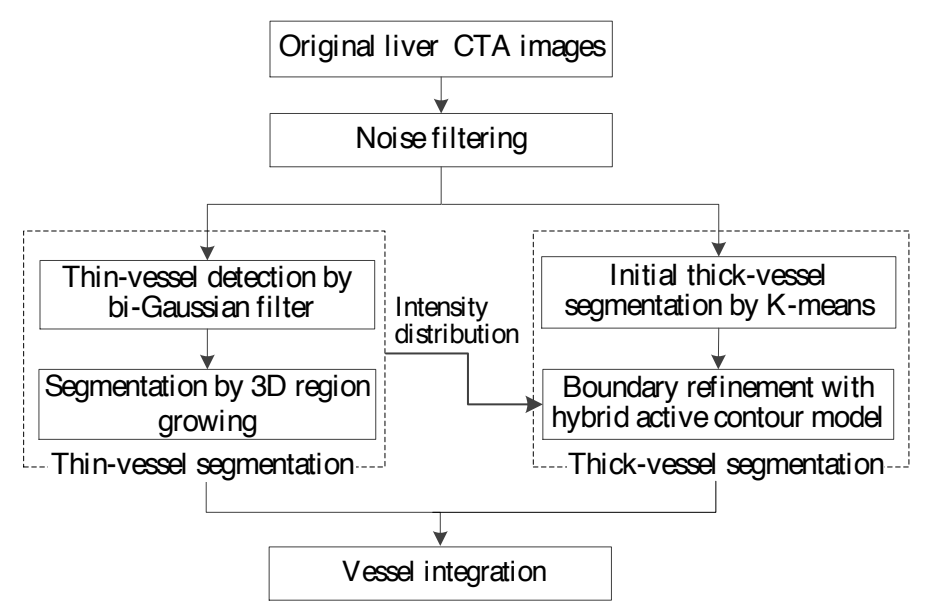

Fig. 1. Flowchart of liver vessel segmentation.

The remainder of this paper is organized as follows. Section 2 describes the details of the proposed method for liver vessel segmentation. In Section 3, the experiments and results are presented. Section 4 gives a discussion, and Section 5 concludes the paper.

\section{Methodology}

Due to the limitation of imaging techniques of CT scans, the CTA images are inevitably contaminated by noise. In addition, to reduce harmful radiation effects of x-rays, the CT scans usually work with low tube voltage, which also leads to the increasing of noise. Therefore, a nonlinear anisotropic diffusion filter described in [12] and [18] is firstly applied to smooth noise while reserving vessel boundaries, then two different strategies are respectively used for the thin and thick vessel segmentation. For the 
anisotropic diffusion filter, the parameter of gradient modulus threshold $\kappa$ is considered as an important sensitive parameter and should be chosen carefully. To obtain an optimal threshold $\kappa$, Fig. 2 shows the MIPs of filtering results with different $x$ s. As observed, a small $\varkappa$ is unable to deal with the liver vessel image contaminated by significant noise, while a large one will lead to a slightly blurring of the vessel boundary. In our experiments, a default value $\kappa=70$ can keep a better balance between noise filter and boundary preserving for significantly noise-corrupted liver vessel images.

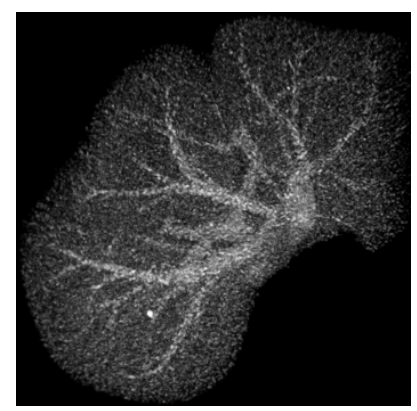

(a)

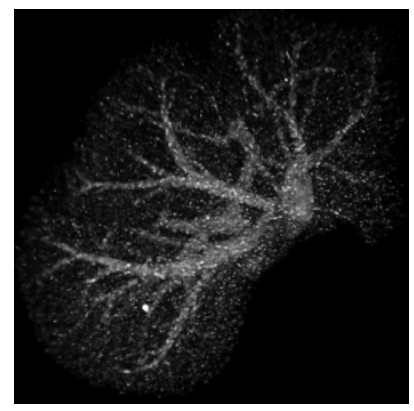

(d)

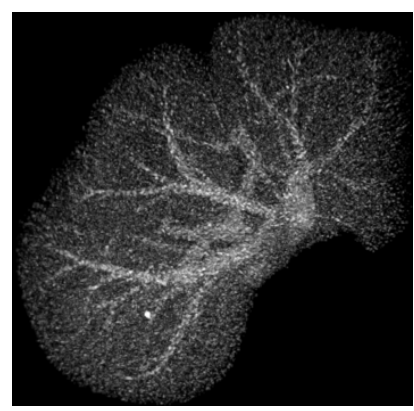

(b)

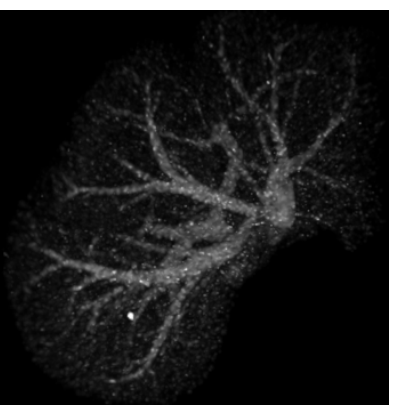

(e)

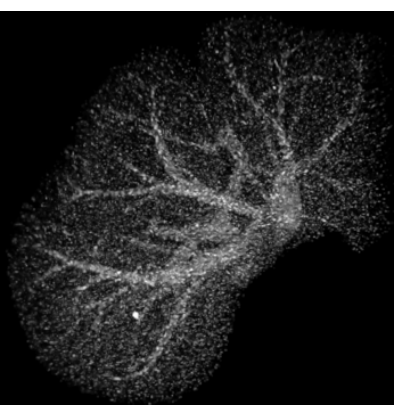

(c)

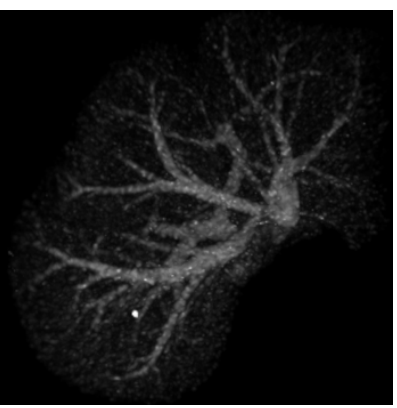

(f)

Fig. 2. Results of anisotropic diffusion filtering with different gradient modulus thresholds. (a) The MIP of original liver vessel image, and (b)-(f) are the MIPs of filtering results with $\kappa$ equal to $10,40,70,80$, and 90 , respectively.

\subsection{Thin vessel segmentation}

Usually, the thin vessels in CTA images suffer from low contrast and blurring edges and therefore, traditional intensity- and gradient- based algorithms, such as the K-means and level set, often fail to segment these vessels. To solve these problems, a thin-vessel segmentation method is proposed based on an analysis of the geometric structure of liver vessels, which first utilizes a bi-Gaussian filter to detect the thin vessels, and then a 3D region growing algorithm for segmentation. 
To detect vessel structure, typical vessel filters usually rely on a scaled Hessian matrix to describe vessel geometry, such as Frangi and medialness filters. As is well known, the Hessian matrix usually involves a convolution operation on the image and second-order derivative of Gaussian kernel [19-21]. However, the Gaussian kernel will lead to the blurring of object boundaries and undesired merging of adjacent objects because of its adopting a single scale on both foreground and background. To deal with the disturbance from adjacent objects, a bi-Gaussian kernel is presented by merging two traditional Gaussian kernels with different scales [19], and is defined as:

$$
B G\left(\sigma, \sigma_{b}, x\right)= \begin{cases}k \cdot G\left(\sigma_{b}, x-\sigma_{b}+\sigma\right), & x \leq-\sigma \\ G(\sigma, x)+\frac{e^{-1 / 2}}{\sqrt{2 \pi}}\left(\frac{\sigma_{b}}{\sigma}-1\right) \frac{1}{\sigma}, & |x|<\sigma \\ k \cdot G\left(\sigma_{b}, x+\sigma_{b}-\sigma\right), & x \geq \sigma\end{cases}
$$

where $\sigma$ and $\sigma_{b}$ are the foreground and background scales, $G(\sigma, x)$ the Gaussian kernel with the mean 0 and variance $\sigma, G\left(\sigma_{b}, x \pm\left(\sigma_{b}-\sigma\right)\right)$ the Gaussian kernels with the mean $\mp\left(\sigma_{b}-\sigma\right)$ and variance $\sigma_{b}$, and $k=\sigma_{b}^{2} / \sigma^{2}$ is set as 0.2 in this paper.

Once the bi-Gaussian kernel is determined, the vessel structure can be described in terms of the eigenvalues of Hessian matrix $H\left(I_{B G}(\sigma, x)\right)$, where $I_{B G}(\sigma, x)$ is the convolution result of $B G\left(\sigma, \sigma_{b}, x\right)$ and $I(x), \quad I_{B G}(\sigma, x)=B G\left(\sigma, \sigma_{b}, x\right) * I(x)$, and $H$ the Hessian matrix. Assuming the eigenvalues $\lambda_{i, \sigma}$ $(i=1,2,3)$ of $H$ are sorted in order, say $\left|\lambda_{1, \sigma}\right| \geq\left|\lambda_{2, \sigma}\right| \geq\left|\lambda_{3, \sigma}\right|$, a bright vessel structure will satisfy $\lambda_{1, \sigma} \approx \lambda_{2, \sigma} \leq 0$ and $\lambda_{3, \sigma} \approx 0$. According to the distribution of eigenvalues, the bi-Gaussian filter [19] is defined as:

$$
V_{B G}(\sigma, x)=\left\{\begin{array}{lc}
-\frac{\lambda_{2, \sigma}}{\lambda_{1, \sigma}} \cdot\left(\lambda_{2, \sigma}+\lambda_{1, \sigma}\right), & \sum_{i=1}^{i=3} \lambda_{i, \sigma}<0 \\
0, & \text { otherwise }
\end{array}\right.
$$

where the ratio of $\lambda_{2, \sigma}$ to $\lambda_{1, \sigma}$ is used to punish deviation from the tubular center, and the sum of the two eigenvalues (i.e., $\lambda_{2, \sigma}$ and $\lambda_{1, \sigma}$ ) defining the vessel cross-section is applied to measure the strength of vessel structure.

Considering the variety of vessel radii, a multi-scale framework is used to obtain the maximum response of vessels with various sizes:

$$
\phi_{B G}(x)=\max \left\{V_{B G}(\sigma, x), \sigma_{\min } \leq \sigma \leq \sigma_{\max }\right\}
$$

where $\sigma_{\min }$ and $\sigma_{\max }$ represent the minimum and maximum scales, respectively, determining the scale 
range of vessels to be detected.

To better describe the impact of scale ranges on vessel detection, Fig. 3 gives the detecting results performed by multi-scale framework, where Fig. 3 (a) is an original liver vessel image obtained by previous works [22, 23], and Figs. 3(b)-(d) show the detecting results of bi-Gaussian filter with different scale ranges $\left[\sigma_{\min }, \sigma_{\max }\right]$ of $[1,2],[1,4]$, and [1, 7], respectively. Here, the visualization of 3D vessel images is implemented in MeVisLab [24], using the volume rendering mode. As observed, a wider scale range of $\left[\sigma_{\min }, \sigma_{\max }\right]$ is beneficial to detect vessels with different scales, but also leads to a relatively weaker response of thin vessels, i.e., a smaller ratio of the intensity of thin vessels to that of thick ones. In this section, as we mainly focus on the detection of thin vessels, the scale range of $\left[\sigma_{\min }, \sigma_{\max }\right]$ is set to a narrow one covering most of the radii of thin vessels, instead of a wide one covering all possible radii of vessels as traditional vessel detection methods do.

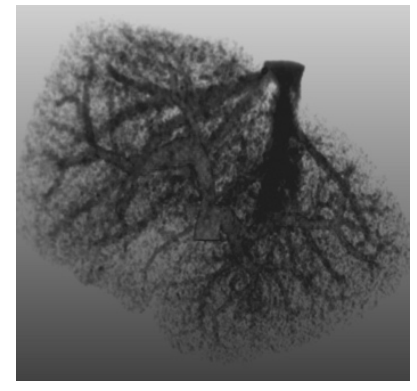

(a)

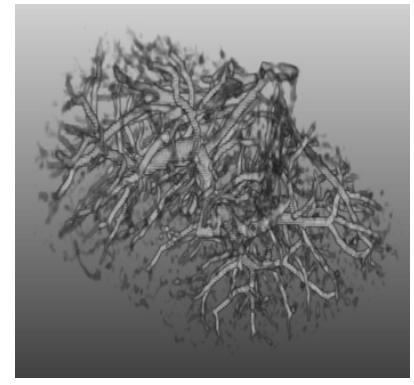

(b)

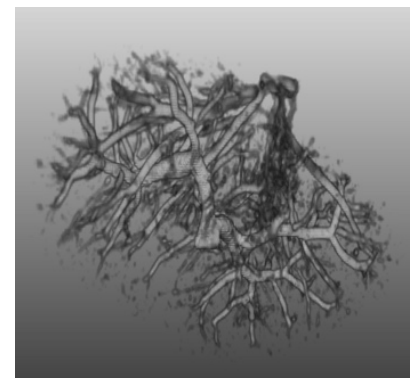

(c)

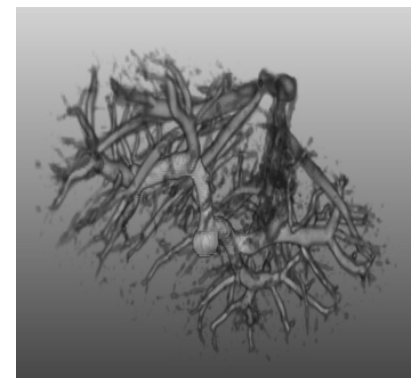

(d)

Fig. 3. Vessel detecting results of bi-Gaussian filter with different scale ranges $\left[\sigma_{\min }, \sigma_{\max }\right]$. (a) Original liver vessels image, and (b) through (d) detetion results with different scale ranges of [1, 2], [1, 4], and [1, 7], respectively.

To efficiently segment thin vessels, a 3D region growing algorithm is then applied on the bi-Gaussian filter detected image. First, the voxels with their intensities ranked in the top $2 \%$ of the whole image are selected as the seed points. Then, if a voxel $x_{s}^{n}$ belonging to the neighborhood of seed voxel $x_{s}$ meets the similarity criterion, $\left|\phi_{B G}\left(x_{s}^{n}\right)-\phi_{B G}\left(x_{s}\right)\right|<t h_{\text {diff }}$ and $\phi_{B G}\left(x_{s}^{n}\right)>t h_{\text {low }}$, it is added to the object and set as a new seed voxel, where the thresholds $t h_{\text {diff }}$ and $t h_{\text {low }}$ are set as 5 and 20 in this paper. The procedure stops until no more voxels satisfy the similarity criterion. In this way, we can obtain thin vessels efficiently. To further analyze the influence of salce range $\left[\sigma_{\min }, \sigma_{\max }\right]$ on segmentation performance, Figs. 4(a)-(c) 
give the corresponding segmentation results of Figs. 3(b)-(d). As seen in Fig. 4, the narrowest scale range $\left[\sigma_{\min }, \sigma_{\max }\right]$ can effectively segment most of the thin vessels, even if some of them suffer from low contrast and blurring boundaries. With the increasing of scale range, the segmentation performance of thin vessels will gradually decrease. Therefore, the scale range $\left[\sigma_{\min }, \sigma_{\max }\right]$ is set as $[1,2]$ for clinical CTA data set, and Fig. 4(a) shows corresponding thin-vessel segmentation result.

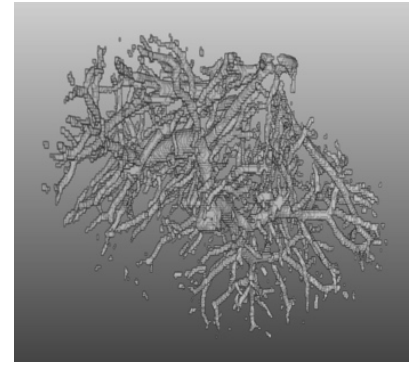

(a)

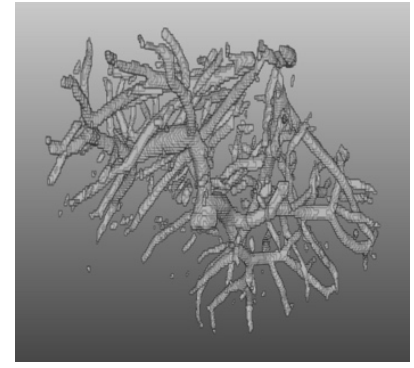

(b)

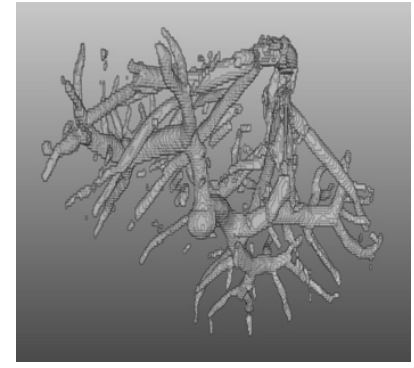

(c)

Fig. 4. Influence of salce range $\left[\sigma_{\min }, \sigma_{\max }\right]$ on 3D region growing. (a)-(c) Segmentation results of Figs. 3(b)-(d), respectively.

\subsection{Thick vessel segmentation}

In this section, a thick-vessel segmentation method is proposed based on K-means clustering and hybrid active contour model. Generally, the active contour model requires an initial segmentation for contour evolution and therefore, a K-means clustering method [25] is performed to generate this initial segmentation. To balance clustering performance and computing time, the cluster number of K-means is set as 3 in our experiments. Figs. 5(a)-(c) show the clustering results of Fig. 3.(a), whose clustering centers are sorted in an ascending order as $2.10,45.90$, and 160.73 , respectively, and they are intuitively corresponding to the background, transition region (from background to thick vessels), and thick vessels, respectively. According to the vessel prior in CTA image, the intensities of the thick vessels are relatively higher than the background and the transition region, and thus we choose the clustering result with maximal clustering center as the initial thick vessels, as shown in Fig. 5(c). 


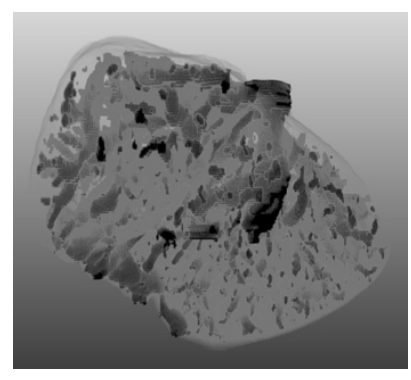

(a)

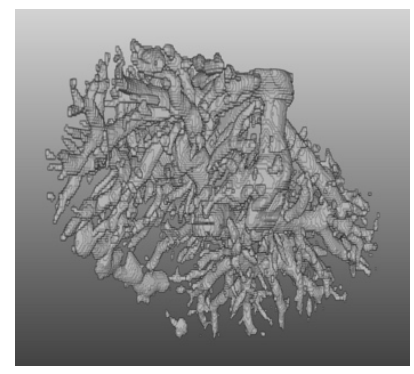

(b)

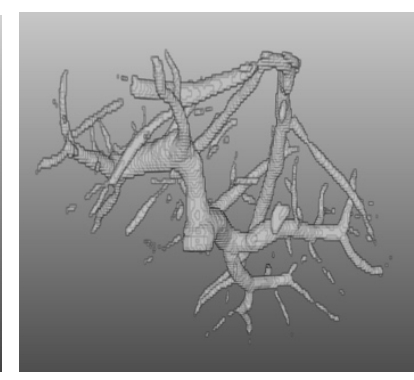

(c)

Fig. 5. K-means clustering results of Fig. 3(a). (a)-(c) Clustering results with clustering centers of 2.10, 45.90 , and 160.73 , respectively.

\subsubsection{Boundary refinement with hybrid active contour model}

Compared with the edge- and region- based active contour model, such as the geodesic active contour (GAC) model and Chan-Vese (CV) model, the hybrid active contour model is more effective to deal with the boundary leakage and intensity inhomogeneity [26]. Therefore, we apply it for the boundary refinement of thick vessels, and its energy function is defined as:

$$
E(\Phi)=-\alpha_{1} \int_{\Omega}(I-\mu) H(\Phi) d \Omega+\alpha_{2} \int_{\Omega} g \mid \nabla H(\Phi) d \Omega
$$

where $\alpha_{1}$ and $\alpha_{2}$ are the region and boundary weight coefficients, respectively, $\Phi$ is the closed contour (or surface in 3D), $\Omega$ the image domain, $\mu$ the lower bound of the intensity in target object, $g=\frac{1}{1+|\nabla I|^{2}}$ is the positive decreasing function, and $H(\Phi)$ is the Heaviside function defined as

$$
H(\Phi)= \begin{cases}0, & \Phi<0 \\ 1, & \Phi \geq 0\end{cases}
$$

According to the theory of gradient descent flow, the partial differential equation (PDE) of the evolution contour is expressed as:

$$
\frac{\partial \Phi}{\partial t}=\delta(\Phi)\left[\alpha_{1}(I-\mu)+\alpha_{2} \operatorname{div}\left(g \frac{\nabla \Phi}{|\nabla \Phi|}\right)\right]
$$

where $\delta(\Phi)$ is a Dirac function with $\delta(\Phi)=d H(\Phi) / d \Phi$.

In Eq. (4), $\mu$ is a key parameter significantly affects the segmentation performance. To give an intuitive understanding, Figs. 6(a)-(c) show the segmentation results of thick liver vessels for Fig. 3(a) with different $\mu$ values of 25,35 , and 60 , respectively. As observed, a smaller $\mu$ value easily leads to serious over-segmentation, as shown in Fig. 6(a), where many intertwined or parallel vessels belonging 
to different vessel systems are mistakenly classified into one vessel system. On the contrary, a larger $\mu$ value results in the loss of many vessel branches, as shown in Fig. 6(c).

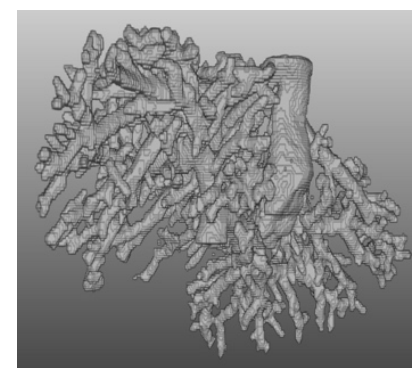

(a)

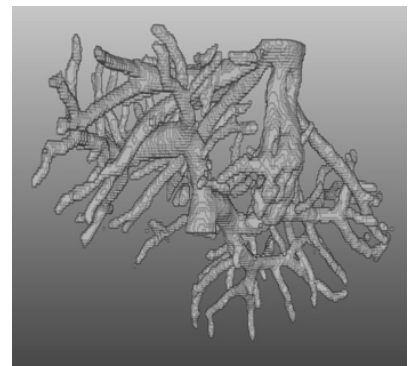

(b)

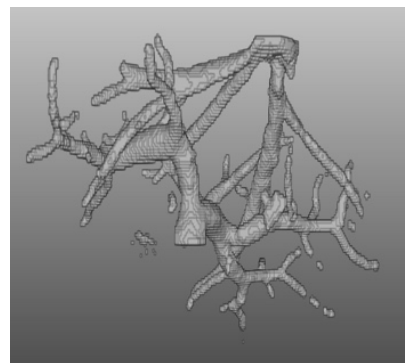

(c)

Fig. 6. Thick liver vessel segmentation results by active contour model with different $\mu$ values of (a) 25 , (b) 35 , and (c) 60 , respectively.

To optimize the parameter $\mu$, vessel prior in CTA images is considered. As stated early, the intensities of the thin vessels are lower than those of the thick vessels, and the similar situation exists between vessel boundaries and their centers. That is, the intensities of thin-vessel boundaries are the lowest among the whole liver vessels, and therefore, they can be used to estimate the parameter $\mu$.

To obtain the intensity information of thin-vessel boundaries, a morphology method is firstly adopted to extract the boundary voxels from thin-vessel segmentation result. Let $A$ be the thin-vessel segmentation result by bi-Gaussian filter combined with 3D region growing, and $\beta(A)$ be the boundary voxels of thin vessels $A, \beta(A)$ can be expressed as

$$
\beta(A)=A-(A \Theta B)
$$

where $\Theta$ represents the erosion operation, and $B$ is a 3D structuring element composed of three $3 \times 3$ matrices in a symmetrical spatial structure, as shown in Fig. 7, in which "0" and "1" are the values of structuring element. As $\beta(A)$ may include noise with high intensity and some non-thin-vessel-boundary voxels, to maximally exclude these voxels, we only consider the voxels in $\beta(A)$ with their intensities in range $\left[I_{\min }, I_{\max }\right]$, and use their mean intensity to specify the parameter $\mu$. By sorting voxels in $\beta(A)$ in an ascending order of their intensities, $I_{\min }$ and $I_{\max }$ are set as the intensities with associated voxels ranked in the top $10 \%$ and top $40 \%$, respectively, i.e., $I_{\min }=I\left(x_{0.1 \cdot N(\beta)}\right)$, and $I_{\max }=I\left(x_{0.4 \cdot N(\beta)}\right)$, where $N(\beta)$ is the voxel number in $\beta(A), x_{0.1 \cdot N(\beta)}$ and $x_{0.4 N(\beta)}$ represent the indices in the ordered 
voxel set.

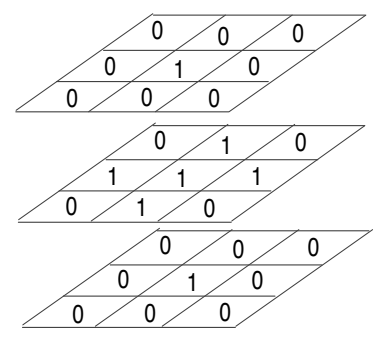

Fig. 7. 3D structuring element for erosion operation. It is composed of three $3 \times 3$ matrices in a symmetrical spatial structure, in which "0" and "1" are the values of structuring element.

Next, a hybrid active contour model is applied to refine the vessel boundaries of thick vessels, in which the vessel segmented by K-means clustering is set as the initial contour and the weigh coefficients $\alpha_{1}$ and $\alpha_{2}$ are respectively set as 0.1 and 0.2 in this paper. Fig. 8 shows an example of boundary refinement, where the first row is the original liver vessel images, and their corresponding boundary refinement results are given in the second row. From Fig. 8, we can find that the proposed method can effectively segment almost all thick vessels, and avoids serious over- or under-segmentation caused by improper $\mu$ values. Moreover, our method can also preserve the smoothness of vessel surfaces and keep the continuity of vessel shapes. However, some thin vessels fail to be segmented due to their lower intensities.
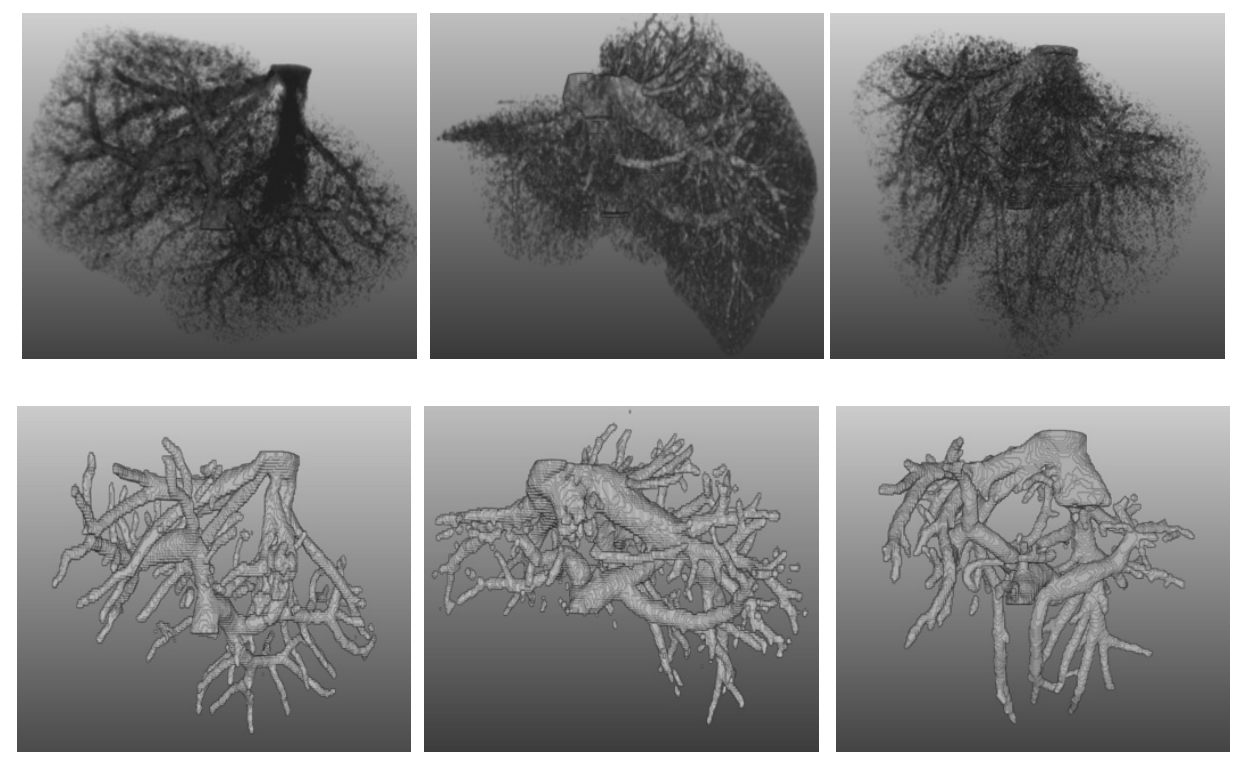
Fig. 8. Thick liver vessel segmentation by hybrid active contour model with $\mu$ obtained by our method. The first row is the original liver vessel images and the second row their corresponding segmentation results.

\subsection{Vessel integration}

As discussed above, the bi-Gaussian filter combined with 3D region growing shows good segmentation results on the thin liver vessels, even if they appear with low contrast and blurring edges, however, not so good segmentation results on the thick liver vessels. On the contrary, the K-means clustering together with active contour model can effectively segment thick vessels, but fails to segment thin vessels with low intensities. Therefore, to exhibit the advantages of these two methods, we integrate their results to obtain a new liver vessel segmentation image. Since the image may contain some isolated regions caused by noise, which are usually incorrectly grouped into the vessels, we reclassify the regions with their volumes less than 20 voxels as the background. An example of liver vessel incorporation as described above is shown in Fig. 9. For intuitive comparison, Figs. 9(a) and (b) repeatedly display the segmentation results of Fig. 3(a), which have been previously shown in Fig. 4(a) and the bottom left of Fig. 8, respectively, and Fig. 9(c) is the final segmentation result which, as observed, is satisfying.

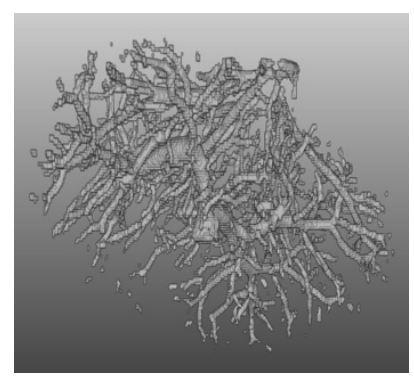

(a)

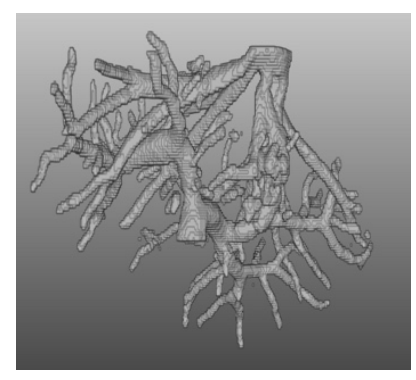

(b)

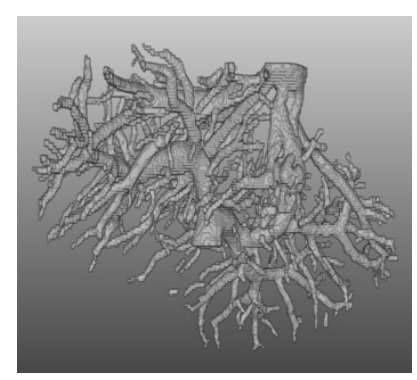

(c)

Fig. 9. Vessel incorporation. (a) Segmentation result of thin vessels, (b) segmentation result of thick vessels, and (c) final segmentation result.

\section{Experiments and Results}

In this section, we will validate the proposed method with a synthetic data set and a clinical CTA data set. The former is generated with the VascuSynth software [27, 28], which includes ten groups of data, 
and each group contains twelve synthetic vessel images with different numbers of bifurcations. The latter includes twelve different patients' CTA volumes at portal venous phase, four of which are pathologic, and each CTA slice has an in-plane resolution of $512 \times 512$ pixels with the slice thicknesses varying from 0.5 to $2 \mathrm{~mm}$.

In the synthetic data set experiments, we normalize the intensities of synthetic images and add Gaussian noise to simulate real CT images. Due to the difference of vessel intensities, shapes, as well as radii between clinical CTA data and synthetic data, we re-optimize some parameters used for the processing of synthetic data such as $\sigma_{\min }=0.9$ and $\sigma_{\max }=1.2$ for thin-vessel detection, and $I_{\min }=I\left(x_{0.55 \cdot N(\beta)}\right)$ and $I_{\max }=I\left(x_{0.65 \cdot N(\beta)}\right)$ for the configuration of parameter $\mu$. In addition, due to lower ratio of vessel volume to whole image volume, the top $0.7 \%$ of voxels with highest intensities are selected as the seed points of $3 \mathrm{D}$ region growing.

Fig. 10 presents the segmentation results of synthetic vessels with different noise levels, where the first row is the MIPs of synthetic images with different Gaussian noises of $\sigma=10,15$, 20, and 25, respectively, and the second row is their corresponding segmentation results. As observed from the first image of the second row in Fig. 10, both of the thin and thick vessels can be effectively segmented from the images with lower noise level. With the increasing of noise, some thin vessels fail to be segmented, but the thick vessels can be still correctly segmented.
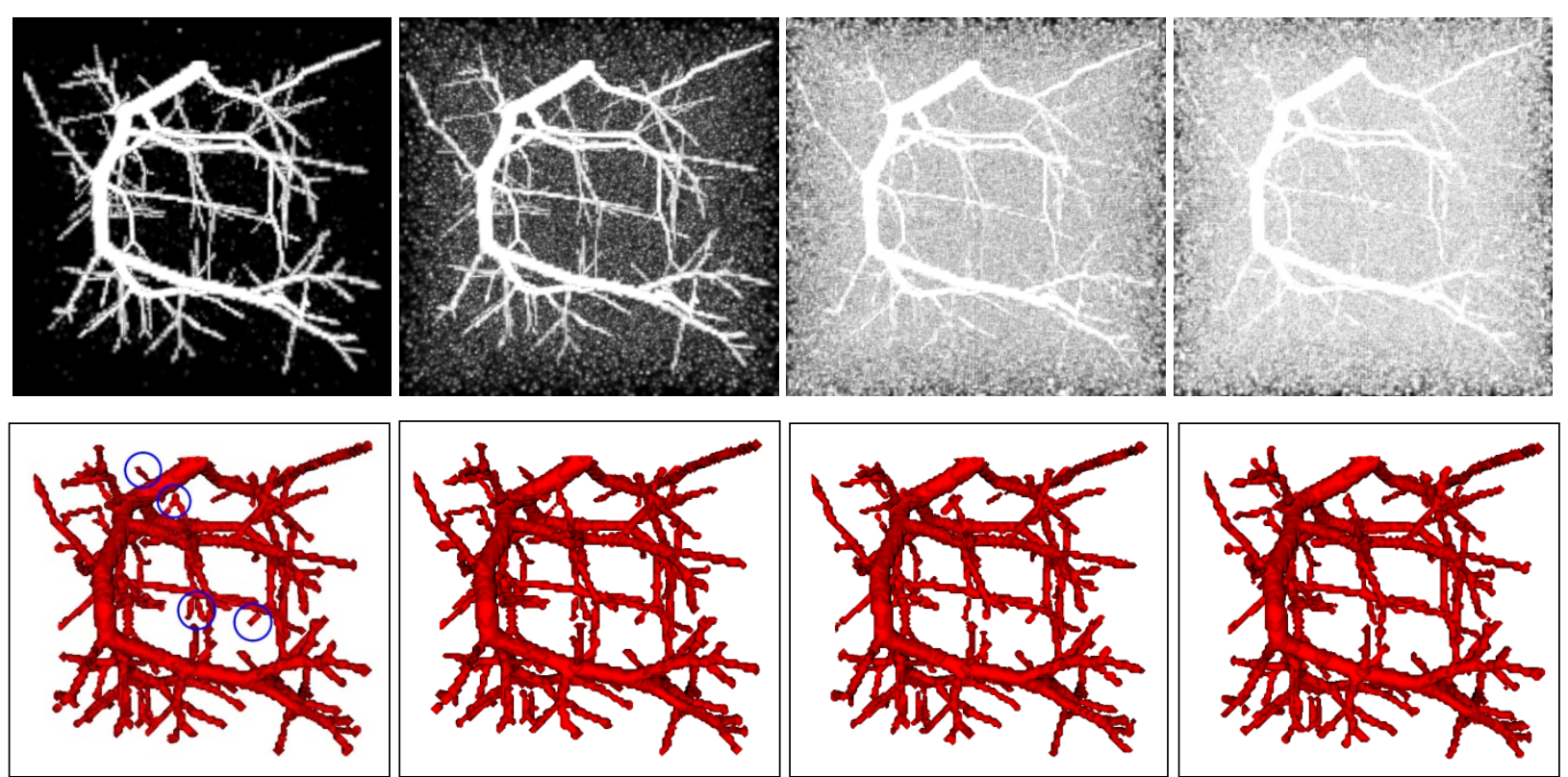

Fig. 10. Segmetnation result of synthetic vessel images with different noise levels. The first row is the MIPs of synthetic images with different Gaussian noises of $\sigma=10,20,30$, and 40, respectively, and the second row is their corresponding segmentation results. The visualization of the color images is 
implemented using Visualization Toolkit (VTK) software (http://www.vtk.org).

To illustrate the effectiveness of the proposed method on different vessel structures, we conduct experiments on these 120 synthetic vessel images with noise level equal to 0.1 , and Fig. 11 shows four typical images and their corresponding segmentation results. As observed, except few vessel branches labeled by blue arrows fail to be extracted, most of the vessels can be accurately segmented. For quantitative analysis, the Dice overlap and Jaccard measures are used to assess the segmentation performance, and our method obtains an average Dice overlap and Jaccard of $86.50 \%$ and $76.31 \%$, respectively, which are very close to those of the recent vessel segmentation methods with average values of $86.76 \%$ and $76.61 \%$ [11]. Thus, we can conclude that the proposed method is an effective automatic vessel segmentation method.
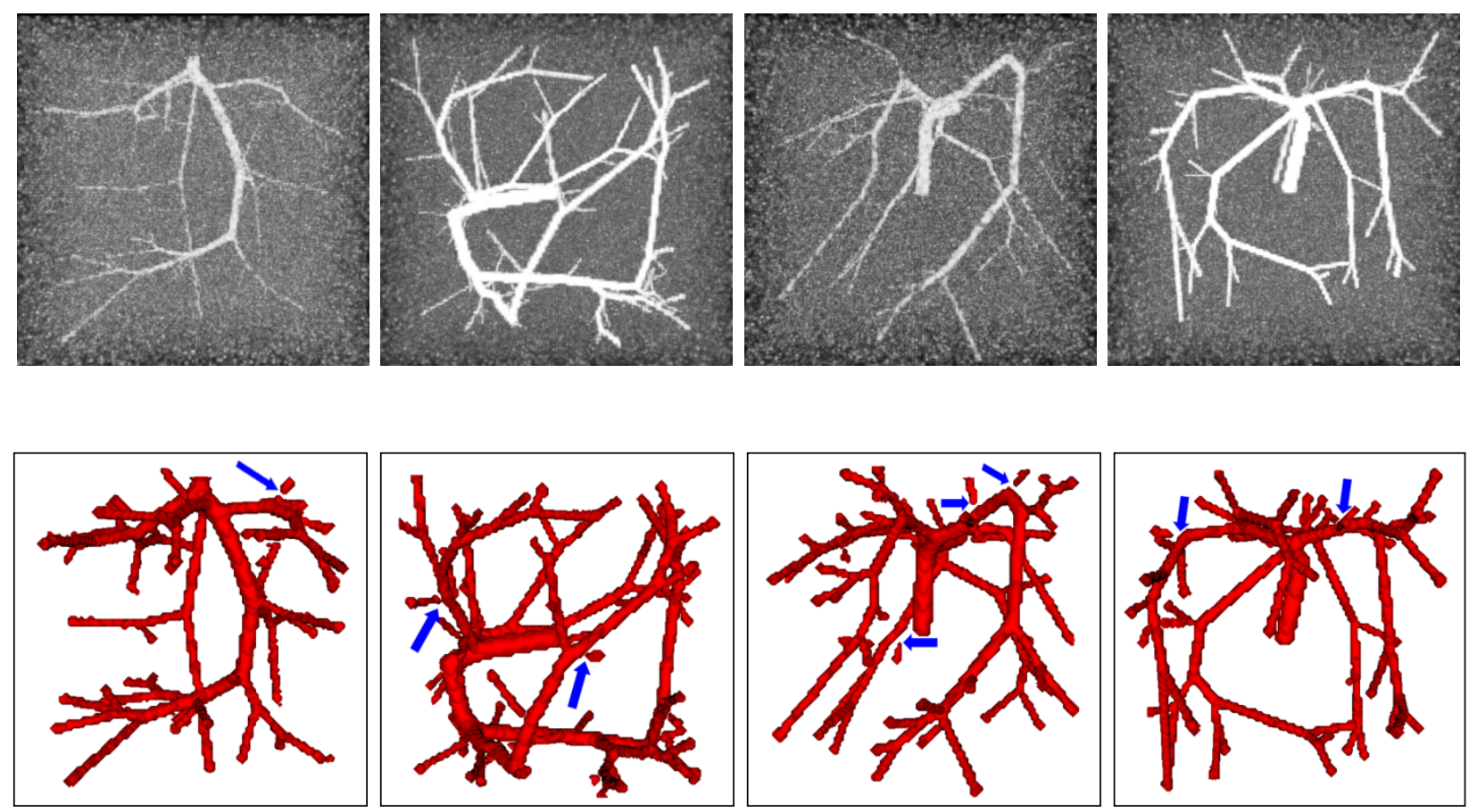

Fig. 11. Segmetnation results of different vessel structures. The first row is the MIPs of synthetic images with noise level equal to 0.1 , and the second row shows their corresponding segmentation results.

For clinical CTA images, the detailed parameters are set as the default values listed in the methodology section. Fig. 12 presents four different original liver vessel images and their corresponding segmentation results by the methods in [14], [29], and the proposed method, respectively. As observed, 
compared with the methods in [14] and [29], the proposed method can segment more thin vessels despite the presence of weak boundaries and low contrast between thin vessels and liver parenchyma, as shown in the closed blue circles in the last row of Fig. 12. This is mainly because the bi-Gaussian filter can effectively highlight thin vessels while suppressing the background by constraining the scale range of multi-scale framework. In addition, due to effective exploitation of both intensity and gradient information of thick vessels, the proposed method also segments the thick vessels more accurately than the other two methods.
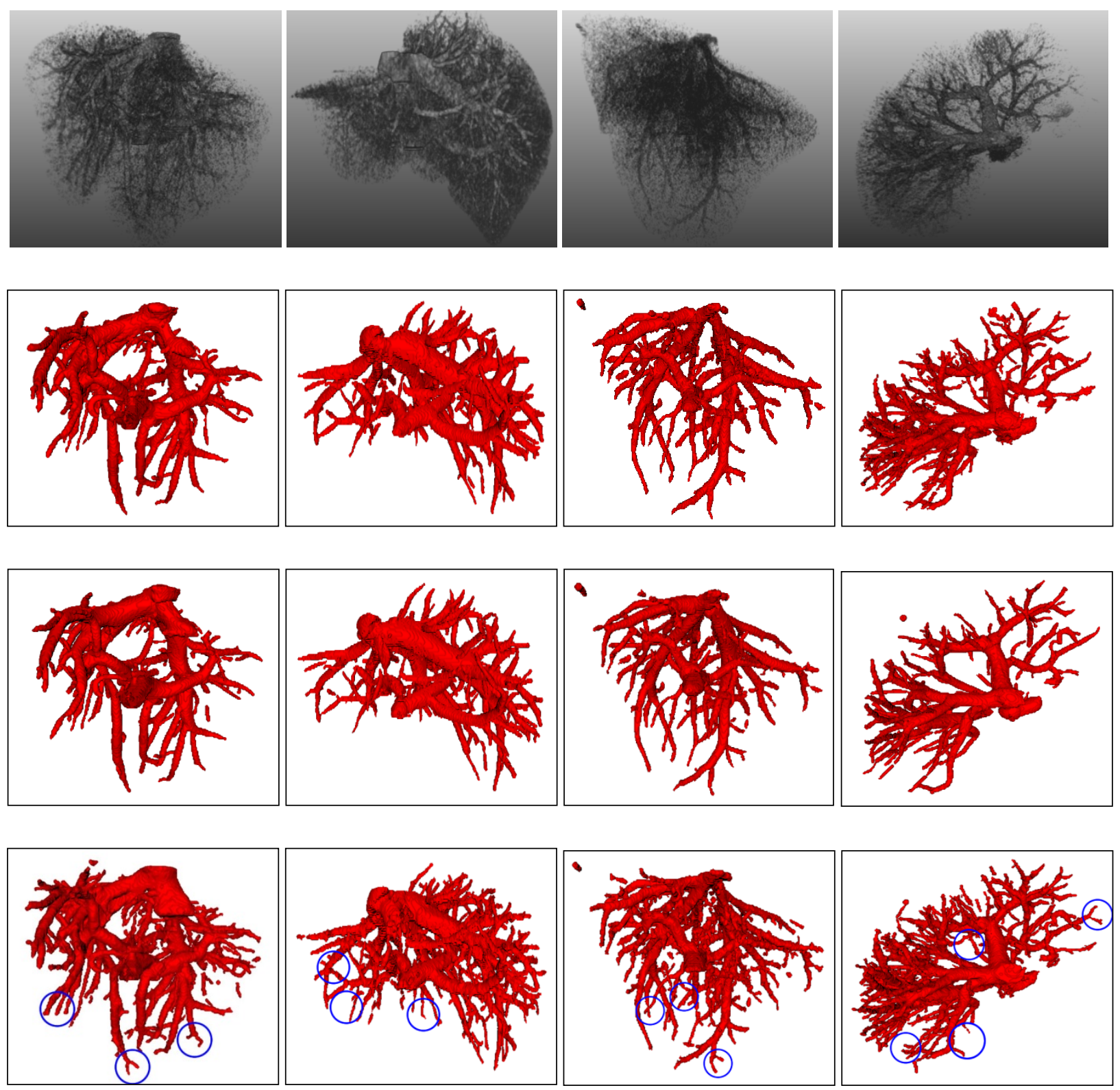

Fig. 12. Liver vessel segmentation results of different methods. The first row is the original liver vessel images, and the second through fourth rows are their corresponding segmentation results by the 
methods in [14], [29], and the proposed method, respectively.

To compare the segmentation performance of the proposed method under the influence of low contrast, an example of liver vessel segmentation is given in Fig. 13, where Figs. 13(a)-(b) are the original liver vessel imges with low contrast, Figs. 13(c)-(d) are their corresponding segmentation results. Compared with the high contrast CTA volumes shown in Fig. 12, the proposed method segments fewer vessel branches due to their obviously lower contrast, but still performs well for the vessels with their intensities slightly higher than those of the background.

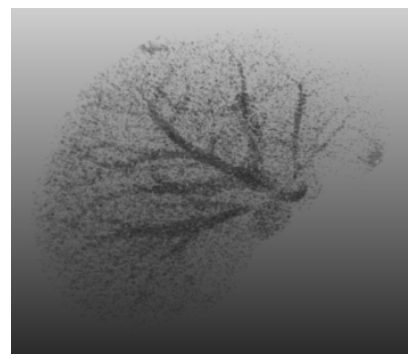

(a)

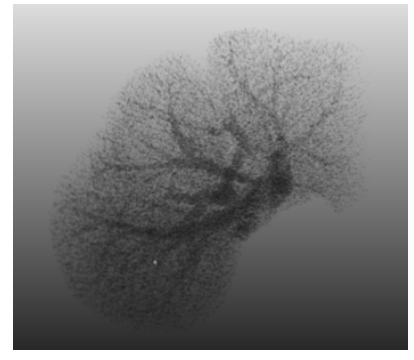

(b)

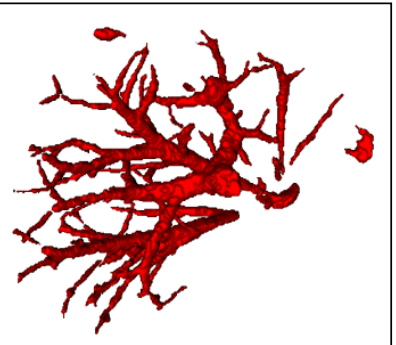

(c)

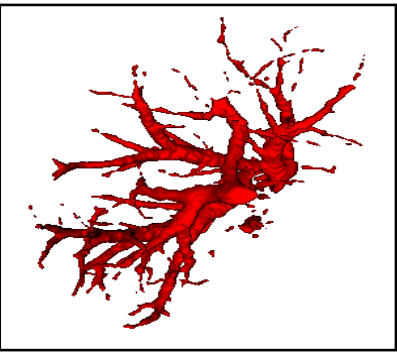

(d)

Fig. 13. Liver vessel segmentation results of low contrast CTA volumes. (a)-(b) Original liver vessel imges with low contrast, and (c)-(d) their corresponding segmentation results.

To further evaluate the segmentation performance, a professional radiologist is invited to guide the generation of the ground truth of liver vessels. First, a trained operator is asked to delineate the initial liver vessels with the help of ITK-SNAP tool (http://www.itksnap.org/pmwiki/pmwiki.php), and then, we correct the delineated results slice by slice under the guidance of the radiologist. Besides Dice overlap and Jaccard, three commonly-used evaluation measurements including accuracy, sensitivity, and specificity, as well as Root Mean Square Symmetric Surface Distance (RMSD) are introduced to indicate the segmentation performance, where RMSD is defined as:

$$
\mathrm{RMSD}=\sqrt{\frac{1}{|S(A)|+|S(B)|}} \times \sqrt{\sum_{p_{A} \in S(A)} d^{2}\left(p_{A}, S(B)\right)+\sum_{p_{B} \in S(B)} d^{2}\left(p_{B}, S(A)\right)}
$$

where $A$ and $B$ represent voxel sets of the segmented and truth liver vessel regions, $S(A)$ and $S(B)$ are the sets of the vessel surface voxels of $A$ and $B$, respectively, $|\bullet|$ is the voxel number, and $d(p, S)$ is the shortest distance of a set $S$. Usually, the lower the RMSD, the better the segmentation result. 
Table 1 shows the segmentation performances on these twelve patients' CTA volumes. It can be found that the average segmentation accuracy, sensitivity, specificity, Dice, Jaccard, and RMSD of our method reach $98.1 \%, 68.3 \%, 99.2 \%, 73.0 \%, 66.1 \%$, and $2.56 \mathrm{~mm}$, respectively. The accuracy and specificity of the proposed method are very close to those of the methods in [14], [29], and [30], whereas our method obtains obviously higher sensitivity, Dice, and Jaccard than the other three methods, which indicates our method is capable of segmenting liver vessels effectively. Note that the average RMSD of our method is a little bigger than that of method in [14]. The reason is that some thin vessels may be ignored by the trained operator due to the low contrast, but they can be segmented by the proposed method, as shown in Fig. 14, which results in longer distances between the surfaces of segmented and truth vessels.

Table 1 Segmentation performances of different methods

\begin{tabular}{ccccccc}
\hline Methods & Accuracy & Sensitivity & Specificity & Dice & Jaccard & RMSD(mm) \\
\hline Method in [14] & 0.980 & 0.677 & 0.994 & 0.670 & 0.603 & 2.27 \\
Method in [29] & 0.976 & 0.657 & 0.992 & 0.645 & 0.554 & 2.72 \\
Method in [30] & 0.970 & 0.501 & 0.994 & 0.540 & 0.430 & 3.05 \\
Our method & 0.981 & 0.683 & 0.992 & 0.730 & 0.661 & 2.56 \\
\hline
\end{tabular}

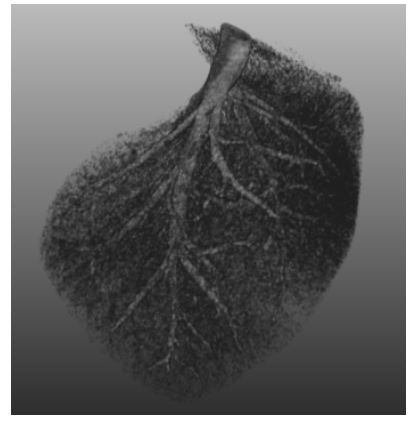

(a)

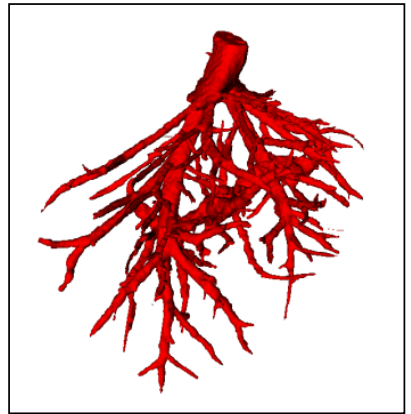

(b)

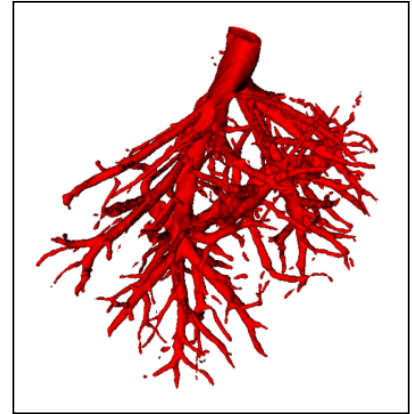

(c)

Fig. 14. Comparison between the ground truth and the segmentation result of the proposed method. (a) Original liver vessels, (b) ground truth, and (c) segmentation result of the proposed method.

There are also some drawbacks in the proposed method. For example, it does not work well for the thick vessels with lower intensities. As shown in the closed region labeled by a white ellipse in Fig. 15(a), part of the thick vessel fail to be segmented (see Fig. 15(b)). This is mainly because the hybrid active contour model tends to segment the vessels with their intensities larger than $\mu$, leading to the fact that the vessels with lower intensities are incorrectly classified into the background. But they can be 
perceived by the radiologist according to the continuity of vessel positions and the intensities between the adjacent slices, and the manual segmentation results are shown in Fig. 15(c).

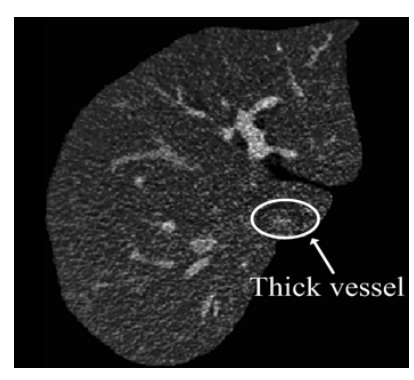

(a)

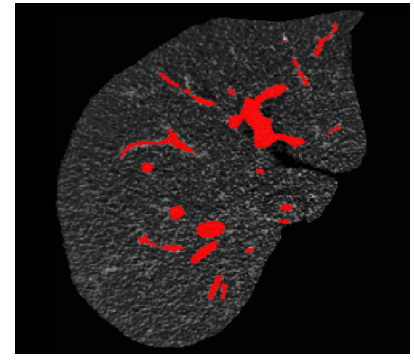

(b)

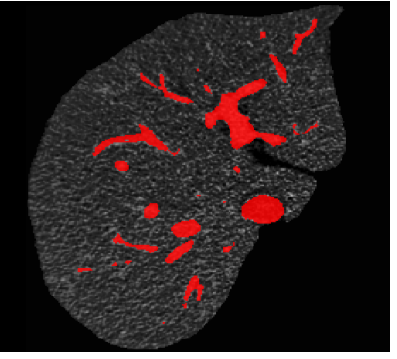

(c)

Fig. 15. Failed segmentation for thick vessel with lower intensities. (a) Original liver slice, (b) vessel segmentation results of the proposed method, and (c) manual segmentation results.

\section{Discussion}

In this paper, we develop a 3D region growing and hybrid active contour model based method for liver vessel segmentation. Generally, due to the intensity inhomogeneity between thin and thick vessels, serious noises as well as the low contrast, an effective segmentation of both thin and thick liver vessels is quite difficult. For example, the GAC model relies on a large propagating coefficient to find more vessel branches, which easily results in over-segmentation. The graph cuts-based methods usually suffer from the problem of shrinking bias on elongated vessel structures, and the same problem also occurs in level set based methods [10]. In fact, due to the complex geometrical structures and intensity distribution, few methods only directly adopt one model or algorithm for the segmentation of 3D vessels, and thus the combination or fusion of two or more methods is necessary and considered $[3,10,11,14]$. Based on this idea, we apply two different strategies to segment both of the thin and thick vessels.

To deal with the thin vessels with low contrast and blurring boundaries, a bi-Gaussian filter is used based on the analysis of vessel structure. Unlike classical vessel detection methods, which usually adopt a wide-scale framework covering all possible radii of vessels to generate the maximum response, a bi-Gaussian filter based on a narrow-scale framework is adopted to highlight these thin vessels. It can partly overcome the weak response of thin vessels during the procedure of multi-scale optimization, as observed in Fig. 3. To avoid manual selection of vessel seeds, an experiential threshold based on vessel prior is performed, followed by a $3 \mathrm{D}$ region growing to automatically segment thin vessels. In the thick-vessel segmentation stage, we first adopt a K-means clustering algorithm to yield an initial vessel 
region, and optimize the parameter $\mu$ according to the vessel intensity and position information. Then, by taking advantages of region and boundary information of CTA volumes, a hybrid active contour model is used to segment thick vessels. In the end, both of thin and thick vessels are intergraded to generate the final liver vessels.

The presented method has been evaluated on a synthetic and a clinical CTA data set, and the experimental results show that it can effectively segment liver vessels, achieving average accuracy, sensitivity, specificity, Dice, Jaccard, and RMSD of $98.1 \%, 68.3 \%, 99.2 \%, 73.0 \%, 66.1 \%$, and $2.56 \mathrm{~mm}$, respectively. In addition, our method can segment more thin vessels compared with the methods in [14], and [29]. Generally, the good performance of the proposed method is mainly ascribed to the effective thin-vessel detection by using a narrow scale framework, as well as optimization of the key parameter $\mu$ of hybrid active contour, which is able to accurately driver the active contour to thick-vessel boundaries.

However, there are also some drawbacks in our method. For example, some thick vessels with lower intensities fail to be segmented, which is also a common problem for other segmentation methods. In addition, the surface of liver vessels segmented by our method is not so smooth compared with some existing methods, although a nonlinear anisotropic diffusion filter is firstly employed to filter image. In the future, we will consider more vessel priors and develop more effective segmentation schemes to increase the robustness of our method.

\section{Conclusions}

This paper presents an effective liver vessel segmentation method based on 3D region growing and hybrid active contour model. As the liver vessels in CTA images usually suffer from low contrast, serious noise, and intensity inhomogeneity between the thin and thick vessels, two different strategies are adopted for effective segmentation of these vessels. For thin liver vessel segmentation, a bi-Gaussian filter is applied to highlight thin vessels based on the selection of narrow scale range in multi-scale framework, followed by a $3 \mathrm{D}$ region growing to segment these vessels. For thick liver vessel segmentation, a K-means clustering algorithm is first applied to generate the initial thick vessels, and a hybrid active contour model is then used for the boundary refinement according to vessel geometric structure and intensity distribution. Finally, both of the thin and thick liver vessel segmentation results are integrated to generate the final liver vessels.

The proposed method does not require any manual selection of seed points, and avoids heavy 
training procedure like the machine learning methods. The experiments on a clinical CTA data set of portal venous phase show that our method achieves better segmentation performance than some other 3D vessel segmentation methods in terms of the accuracy, sensitivity, specificity, Dice, Jaccard, and RMSD. In addition, the segmentation results of the proposed method contain more thin vessels with continuous and complete details, even if some of them appear with low contrast and blurring boundaries, which are very difficult to be segmented by traditional algorithms. Moreover, our method can also accurately segment thick vessels by employing both of their intensity and gradient information.

\section{Acknowledgments}

This work is supported by the National Natural Science Foundation of China (Grant nos. 61772555, 61379107, 61772556, 61172184, and 61702179), China Postdoctoral Science Foundation (Grant no. 2012M521554), Program for Hunan Province Science and Technology Basic Construction (Grant no. 20131199), Hunan Provincial Natural Science Foundation of China (Grant no. 2017JJ3091), and Scientific Research Fund of Hunan Provincial Education Department (Grant no.17C0645).

The authors would like to acknowledge radiological experts Drs. Zhen Yang and Tie-xiang Lai for their valuable suggestion and the ground truth establishment of liver vessels, and thank the anonymous reviewers for their valuable suggestions.

\section{References}

[1] D. Selle, B. Preim, A. Schenk, H. O. Peitgen, Analysis of vasculature for liver surgical planning, IEEE Trans. Med. Imaging. 21 (11) (2002) 1344-1357.

[2] C. Y. Xiao, M. Staring, D. Shamonin, J. H. C. Reiber, J. Stolk, B. C. Stoel, A strain energy filter for 3D vessel enhancement with application to pulmonary CT images, Med. Image Anal. 15 (1) (2011) 112-124.

[3] Y. Shang, R. Deklerck, E. Nyssen, A. Markova, M. J. De, X. Yang, K. Sun, Vascular active contour for vessel tree segmentation, IEEE Trans. Biomed. Eng. 58 (4) (2011) 1023-1032.

[4] Y. Cheng, X. Hu, J. Wang, Y. Wang, S. Tamura, Accurate Vessel Segmentation with Constrained B-snake, IEEE Trans. Image Process. 24 (8) (2015) 2440-2455. 
[5] T. Woźniak, M. Strzelecki, A. Majos, L. Stefańczyk, 3D vascular tree segmentation using a multiscale vesselness function and a level set approach, Biocybern. Biomed. Eng. 37 (2017) 66-77.

[6] S. Lu, H. Huang, P. Liang, G. Chen, L. Xiao, Hepatic vessel segmentation using variational level set combined with non-local robust statistics, Magn. Reson. Imaging. 36 (2016) 180-186.

[7] S. Zhao, M. Zhou, Y. Tian, P. Xu, Z. Wu, Q. Deng, Extraction of vessel networks based on multiview projection and phase field model, Neurocomputing. 162 (C) (2015) 234-244.

[8] X. Gao, Y. Uchiyama, X. Zhou, T. Hara, T. Asano, H. Fujita, A Fast and Fully Automatic Method for Cerebrovascular Segmentation on Time-of-Flight (TOF) MRA Image, J. Digit. Imaging. 24 (4) (2011) 609-625.

[9] C. Bauer, T. Pock, E. Sorantin, H. Bischof, R. Beichel, Segmentation of interwoven 3d tubular tree structures utilizing shape priors and graph cuts, Med. Image Anal. 14 (2) (2010) 172-184.

[10]S. Esneault, C. Lafon, J. L. Dillenseger, Liver vessels segmentation using a hybrid geometrical moments/graph cuts method, IEEE Trans. Biomed. Eng. 57 (2) (2010) 276-283.

[11] N. Sangsefidi, A. H. Foruzan, A. Dolati, Balancing the data term of graph-cuts algorithm to improve segmentation of hepatic vascular structures, Comput. Biol. Med. 93 (2017) 117-126.

[12]Y. Z. Zeng, Y. Q. Zhao, P. Tang, M. Liao, Y. X. Liang, S. H. Liao, B. J. Zou, Liver vessel segmentation and identification based on oriented flux symmetry and graph cuts, Comput. Meth. Programs Biomed. 150 (2017) 31-39.

[13]M. W. Law, A. C. Chung, A deformable surface model for vascular segmentation, in: Med. Image Comput. Comput.-Assist. Interv., 2009, pp. 59-67.

[14]Y. Z. Zeng, Y. Q. Zhao, M. Liao, B. J. Zou, X. F. Wang, W. Wang, Liver vessel segmentation based on extreme learning machine, Phys. Medica. 32 (5) (2016) 709-716.

[15]A. H. Foruzan, R. A. Zoroofi, Y. Sato, M. Hori, A Hessian-based filter for vascular segmentation of noisy 
hepatic CT scans, Int. J. Comput. Assist. Radiol. Surg. 7 (2) (2012) 199-205.

[16]X. Yang, J. D. Yang, P. H. Hong, H. C. Yu, S. Ahn, B. W. Kim, H. You, Segmentation of liver and vessels from CT images and classification of liver segments for preoperative liver surgical planning in living donor liver transplantation, Comput. Meth. Programs Biomed. 158 (2017) 41-52.

[17]M. Marcan, D. Pavliha, M. M. Music, I. Fuckan, R. Magjarevic, D. Miklavcic, Segmentation of hepatic vessels from MRI images for planning of electroporation-based treatments in the liver, Radiol. Oncol. 48 (3) (2014) $267-281$.

[18]P. Perona, J. Malik, Scale-space and edge detection using anisotropic diffusion, IEEE Trans. Pattern Anal. Mach. Intell. 12 (7) (1990) 629-639.

[19] C. Xiao, M. Staring, Y. Wang, D. P. Shamonin, B. C. Stoel, Multiscale bi-Gaussian filter for adjacent curvilinear structures detection with application to vasculature images, IEEE Trans. Image Process. 22 (1) (2012) 174-188.

[20]K. Krissian, G. Malandain, N. Ayache, R. Vaillant, Y. Trousset, Model-Based Detection of Tubular Structures in 3D Images, Comput. Vis. Image Underst. 80 (2) (1999) 130-171.

[21]A. Frangi, W. Niessen, K. Vincken, M. Viergever, Multiscale vessel enhancement filtering, in: Med. Image Comput. Comput.-Assist. Interv., 1998, pp. 130-137.

[22]T. Heimann, G. B. Van, M. A. Styner, Y. Arzhaeva, V. Aurich, C. Bauer, A. Beck, C. Becker, R. Beichel, G. Bekes, Comparison and evaluation of methods for liver segmentation from CT datasets, IEEE Trans. Med. Imaging. 28 (8) (2009) 1251-1265.

[23]M. Liao, Y. Q. Zhao, X. Y. Liu, Y. Z. Zeng, B. J. Zou, X. F. Wang, F. Y. Shih, Automatic liver segmentation from abdominal CT volumes using graph cuts and border marching, Comput. Meth. Programs Biomed. $143(2017) 1-12$.

[24]I. Bitter, U. R. Van, I. Wolf, L. Ibáñez, J. M. Kuhnigk, Comparison of four freely available frameworks for 
image processing and visualization that use ITK, IEEE Trans. Vis. Comput. Graph. 13 (3) (2007) 483-493.

[25]E. Lee, M. Schmidt, J. Wright, Improved and simplified inapproximability for K-means, Inf. Process. Lett. 120 (2017) 40-43.

[26]Y. Zhang, B. J. Matuszewski, L. K. Shark, C. J. Moore, Medical Image Segmentation Using New Hybrid Level-Set Method, in: BioMedical Visualization, 2008. MEDIVIS '08. Fifth International Conference, 2008, pp. 71-76.

[27]G. Hamarneh, P. Jassi, VascuSynth: simulating vascular trees for generating volumetric image data with ground-truth segmentation and tree analysis, Comput. Med. Imaging Graph. 34 (8) (2010) 605-616.

[28]P. Jassi, G. Hamarneh, VascuSynth: Vascular Tree Synthesis Software, Insight J. January-June (2011) $1-12$.

[29]F. Conversano, R. Franchini, C. Demitri, L. Massoptier, F. Montagna, A. Maffezzoli, A. Malvasi, S. Casciaro, Hepatic vessel segmentation for 3D planning of liver surgery experimental evaluation of a new fully automatic algorithm, Acad. Radiol. 18 (4) (2011) 461-470.

[30]E. Smistad, A. C. Elster, F. Lindseth, GPU accelerated segmentation and centerline extraction of tubular structures from medical images, Int. J. Comput. Assist. Radiol. Surg. 9 (4) (2014) 561-575. 